\title{
Papel das áreas de conservação no bem-estar das comunidades locais: caso da Reserva Nacional de Niassa
}

Devido à insustentabilidade da exploração de recursos naturais na África, houve a introdução de alterações nas formas de gestão das áreas de conservação, e que trouxeram consigo um debate sobre o impacto social dos programas de conservação. Com o objetivo de analisar o papel da reserva Nacional do Niassa no bemestar das comunidades locais, foi realizado um trabalho em dois postos administrativos da Reserva Nacional do Niassa, os postos administrativos de Mecula-Sede e Lugenda. Foram entrevistadas 136 pessoas dentre membros da comunidade, líderes locais e colaboradores da Reserva Nacional do Niassa e após as entrevistas os dados coletados foram submetidos a uma análise descritiva para obtenção de tabelas de percentagem, teste chi-quadrado de independência e à Regressão de Poisson para verificar a relação entre variáveis. Os resultados revelam maior participação dos homens nas atividades de maneio e conservação em relação às mulheres. Diferenças significativas foram encontradas na maneira como é são aproveitados os benefícios nos dois postos administrativos estudados. Maior parte dos benefícios da conservação são pouco perceptíveis para alguns dos membros da comunidade pois maiores beneficiados são os indivíduos detentores de poder dentro da comunidade.

\section{Role of conservation areas in the well-being of local communities: case of Niassa National Reserve}

\begin{abstract}
Due to the unsustainability of the exploitation of natural resources in Africa, changes have been made in the management of conservation areas, bringing with it a debate on the social impact of conservation programs. In order to analyze the role of the Niassa National Reserve in the well-being of local communities, work was carried out in two administrative posts of the Niassa National Reserve, the administrative posts of Mecula Headquarters and Lugenda. 136 people were interviewed from community members, local leaders and collaborators of the Niassa National Reserve and after the interviews the collected data were submitted to a descriptive analysis to obtain percentage tables, chi-square independence test and Poisson Regression for check the relationship between variables. The results reveal greater participation of men in management and conservation activities compared to women. Significant differences were found in the way the benefits are harnessed at the two administrative posts studied. Most of the benefits of conservation are barely noticeable to some of the community members as the greatest beneficiaries are the empowered individuals within the community.
\end{abstract}

Keywords: Conservation; Communities; Welfare.

Topic: Gestão de Unidades de Conservação

Reviewed anonymously in the process of blind peer.

Jonas Zefanais Massuque (iD

Universidade Lúrio, Moçambique

http://lattes.cnpq.br/7934340314867218

http://orcid.org/0000-0001-6162-0287

massuquejonas@gmail.com

Merlindo Jacinto Manjate (ib

Universidade Lúrio, Moçambique

http://lattes.cnpq.br/6067118027738336

http://orcid.org/0000-0001-9574-9687

merlindomanjate@unilurio.ac.mz

Custódio Efraim Matavel

Universidade Lúrio, Moçambique

http://lattes.cnpq.br/8420906860220090

http://orcid.org/0000-0002-3800-7887

custodiomatavel@gmail.com
Received: 20/01/2019

Approved: 28/04/2019
6

DOI: 10.6008/CBPC2318-2881.2019.001.0008
Referencing this:

MASSUQUE, J. Z.; MANJATE, M. J.; MATAVEL, C. E.. Papel das áreas de conservação no bem-estar das comunidades locais: caso da Reserva Nacional de Niassa. Nature and Conservation, v.12, n.1, p.75-82, 2019. DOI: http://doi.org/10.6008/CBPC2318-2881.2019.001.0008 


\section{INTRODUÇÃO}

A preocupação pela preservação dos recursos naturais tem sido cada vez mais crescente nas últimas décadas. Dada a insustentabilidade da exploração dos recursos naturais em África, houve a introdução de alterações nas formas de gestão das áreas de conservação, bem como nas áreas comunitárias, detentoras de um potencial de recursos naturais (MATOS et al., 2010). Estas alterações trouxeram consigo um debate sobre o impacto social dos programas de conservação (ADAMS, 2004), uma vez que a conservação contínua tem trazido benefícios económicos inferiores aos observados quando se explora os recursos (BALMFORD, 2002), e muitas vezes há diferenças entre as comunidades locais e as agências de conservação no que respeita aos motivos que levam à conservação (FERNANDES-BACA et al., 2007).

As comunidades esperam que as agências de conservação tenham sempre em conta os padrões do uso dos recursos e os benefícios do uso destes para a sua sobrevivência, fato que muitas das vezes não se tem verificado (GRIFFITHS, 2005; SILVA, 2000; SCHERL, 2006). Assim, as áreas de conservação deverão promover ações que contribuam para a redução da pobreza a nível local por forma a garantir maior envolvimento das comunidades no processo de conservação (STEARMAN, 1996).

Moçambique é um país cujo índice de pobreza é cerca de $50 \%$ e a maioria da população (mais de 70\%) vive nas áreas rurais (ORAM, 2010) tendo como base de subsistência, a agricultura, caça, extração de lenha e carvão. Neste sentido, para garantir um maneio efetivo e sustentável dos recursos, foi introduzido o Manejo Comunitário dos Recursos Naturais (MATOS et al., 2010), com vista a permitir maior participação dos utentes (comunidades locais) na gestão dos recursos naturais de forma que estes possam colher benefícios e garantir o seu uso sustentável (DISTA et al., 2009; NHANTUMBO et al., 2003).

Ademais, de acordo com Diploma Ministerial n093/2005, de 4 de maio, dos Ministérios da Agricultura, Turismo e das Finanças, ao abrigo da Lei no10/99 de 7 de Junho, do Decreto nำ27/2003, de 17 de Junho, do Diploma Ministerial 92/95, de 12 de Julho, do Diploma Legislativo no2629, de 7 de Agosto de 1965 as comunidades que vivem dentro dos parques e reservas nacionais tem direito a $20 \%$ dos benefícios da utilização dos recursos florestais e faunísticos, bem como do exercício do turismo.

Todavia, a aplicação deste instrumento econômico de gestão ambiental tem vindo a enfrentar diversos constrangimentos, o que compromete o aproveitamento destes benefícios por parte das comunidades. Dentre os diversos constrangimentos, destacam-se os atrasos no desembolso dos $20 \%$ e predominância de interesses políticos na comunidade, dificultando por parte das comunidades, em reunir os requisitos previstos no Diploma Ministerial no93/2005 para que tenham acesso aos fundos de 20\% (MICOA, 2014), fraco envolvimento dos membros das comunidades beneficiárias (especialmente mulheres) na planificação dos projetos uma vez que ela é realizada fundamentalmente pelos membros dos Conselhos de Gestão Local (CGL) e líderes comunitários (PRODEZA, 2009).

Além dos $20 \%$, a criação de postos de emprego tem sido apontada, principalmente nos discursos políticos, como um dos benefícios resultantes das áreas de conservação. Contudo, são escassos estudos que trazem uma abordagem quantitativa que mostram tal facto. Ademais, a província do Niassa foi apontada 
pelo Ministério da Agricultura, em 2012, como uma das províncias que menos se beneficiou dos $20 \%$ da exploração dos seus recursos (MINAG, 2012), apesar da mesma possuir uma reserva com vários aglomerados populacionais e duas sedes distritais. Pretende-se, com o presente trabalho, analisar o papel da reserva Nacional do Niassa no bem-estar das comunidades locais.

\section{METODOLOGIA}

\section{Área de estudo}

O trabalho foi realizado em dois postos administrativos da Reserva Nacional do Niassa, os postos administrativos de Mecula-Sede e Lugenda. Esta reserva localiza-se a Norte de Moçambique, cobre uma extensão de aproximadamente $42000 \mathrm{~km}^{2}$, possui uma área central $\left(23040 \mathrm{~km}^{2}\right)$ e uma zona tampão dividida em cinco blocos de caça $\left(19239 \mathrm{~km}^{2}\right)$. O seu limite a norte é o Rio Rovuma, que também serve de fronteira com a Tanzânia. A reserva abrange na totalidade ou em parte oito distritos em duas províncias, nomeadamente Niassa (Mecula, Mavago, Marrupa, Majune, Muembe e Sanga) e Cabo Delgado (Mueda e Montepuez).

Os distritos de Mavago e Mecula estão completamente contidos na Reserva. Possuem vegetação tipicamente de miombo e uma grande riqueza faunística, que compreende 13000 elefantes, 13000 pala-palas e 6000 búfalos. Existe ainda um número de subespécies endémicas com destaque para impala Johnson, o cocone Nyasa e a zebra Bohm. Ainda nesta reserva, existem perto de 450 espécies de pássaros, 50 espécies de cobras, 20 à 30 espécies de lagartos, assim como 23 espécies de tartarugas, cágados e crocodilos do Nilo, cerca de 30 espécies de peixes, muitas das quais com valor comercial e usadas para fins de subsistência (NHONGO, 2017).

\section{Coleta de dados}

Para a coleta de dados foram entrevistadas 136 pessoas dentre membros da comunidade, líderes locais e colaboradores da Reserva Nacional do Niassa, conforme ilustra a tabela 1. A entrevista foi feita com base num guião previamente elaborado e com auxílio de um guia de campo. Os dados coletados incluíram as características socioeconômicas dos residentes na reserva (idade, sexo, tamanho do agregado familiar, fontes de renda/sobrevivência), o papel da comunidade na Gestão de recursos, as práticas usadas na conservação de recursos e o destino dos $20 \%$ do valor resultante da exploração dos recursos alocados. Adicionalmente foi usada Análise Documental que permitiu a consulta do Plano de Maneio da Reserva, relatórios elaborados pela Reserva e legislação Moçambicana sobre a matéria em estudo.

Tabela 1: Número de entrevistados.

\begin{tabular}{|l|c|l|}
\hline Entrevistados & Número de entrevistados & Observação \\
\hline Administração da Reserva & 1 & Administrador da Reserva \\
\hline Governo do Distrito & 1 & Diretor dos Serviços distritais de Atividades Económicas (SDAE) \\
\hline Autoridade tradicional & 2 & Régulo da localidade de Lugenda e chefe tribal da Sede do distrito de Mecula \\
\hline Comités de gestão & 2 & Secretários da localidade de Mecula e Lugenda \\
\hline Pessoas singulares & 130 & 85 da localidade de Lugenda e 45 da localidade de Mecula \\
\hline Total & 136 & Número total dos indivíduos entrevistados \\
\hline
\end{tabular}




\section{Análise dos dados}

Após a coleta, os dados foram submetidos a uma análise descritiva, tendo-se obtido os respectivos gráficos e tabelas de percentagem. O teste chi-quadrado de independência foi usado para verificar a relação entre as atividades de maneio e conservação a que estão envolvidos os membros da comunidade e a localidade em que residem. As possíveis associações entre o número de atividades de maneio e conservação a que cada entrevistado está envolvido e as características socioeconômicas foram verificadas por meio de Regressão de Poisson (BATISTA, 2015), empregando o programa estatístico R (versão 3.4.1), sendo que foi feito o teste de superdispersão e não houve evidências de existência de superdispersão dos dados ( $p$. value $=0.99, \mathrm{c}=0.34)$.

\section{RESULTADOS}

\section{Caracterização dos entrevistados}

De acordo com a figura 1 , nos dois Postos Administrativos os indivíduos entrevistados, na sua maioria tem idades compreendidas entre os 18 e 42 anos, o que revela uma tendência de a população ser predominantemente jovem. Quanto às atividades de subsistência, a agricultura ocupa o primeiro lugar, sendo praticada por mais de $90 \%$ dos indivíduos entrevistados e poucas pessoas estão envolvidas nas atividades comerciais (cerca de 10\% em Mecula-Sede e 5\% em Lugenda), o que revela grande dependência da população dos dois postos administrativos na agricultura.
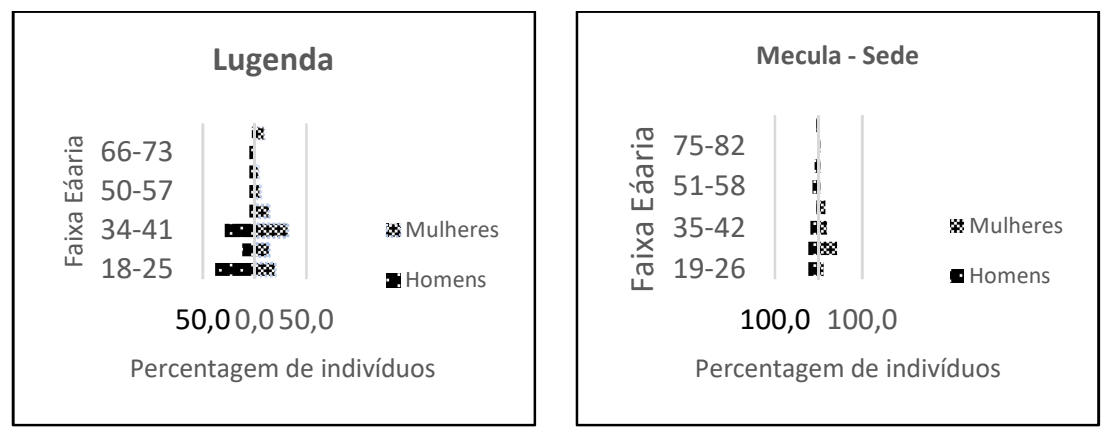

Figura 1: Distribuição Etária dos entrevistados.

Em relação às práticas de maneio e conservação, todos os entrevistados revelaram estar envolvidos no controlo de queimadas descontroladas, o não abate de animais é também um dos meios de conservação praticados pela maioria dos entrevistados (95\% em Mecula-Sede e 93\% em Lugenda).A maior parte dos praticantes da pesca não tem observado as boas práticas de conservação, sendo que a situação é mais crítica no posto administrativo de Mecula, dado que apenas $3 \%$ dos entrevistados não usam venenos para a prática da pesca. Ademais, os resultados do teste de Independência revelam a existência de relação entre o tipo de medida de conservação e Maneio e o Posto administrativo em que residem os entrevistados $\left(P_{\text {value }}=0.00\right.$, $\mathrm{GL}=7, \mathrm{Xcal}=31.3$ ). 


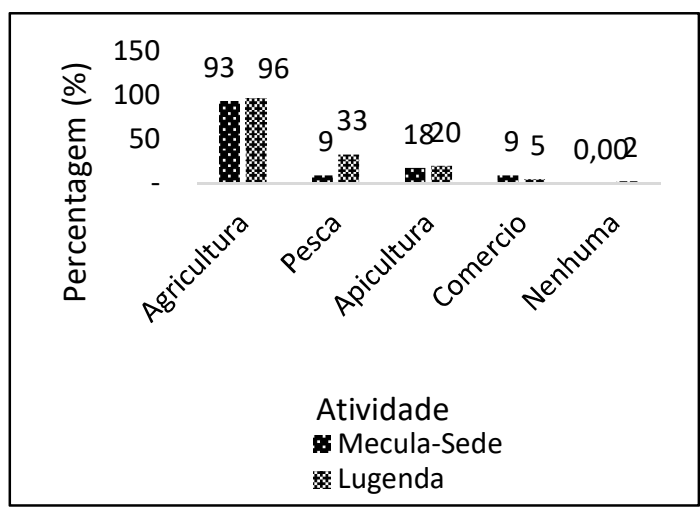

Figura 2: Principais atividades de subsistência.

Tabela 1: Práticas de Maneio e Conservação.

\begin{tabular}{|c|c|c|}
\hline \multirow{2}{*}{ Medida de Conservação } & \multicolumn{2}{|c|}{ Posto Administrativo } \\
\cline { 2 - 3 } & Mecula & Lugenda \\
\hline Controle de queimadas & $100 \%$ & $100 \%$ \\
\hline Controlo da caça ilegal & $84 \%$ & $71 \%$ \\
\hline Licenciamento para pesca & $53 \%$ & $53 \%$ \\
\hline Não abater animais & $25 \%$ & $24 \%$ \\
\hline Não abater árvores de espécies protegidas & $84 \%$ & $35 \%$ \\
\hline Não usar redes finas na pesca & $4 \%$ & $33 \%$ \\
\hline Não usar veneno na pesca & $4 \%$ & $33 \%$ \\
\hline
\end{tabular}

Quanto aos benefícios, a maior parte dos entrevistados apontam o apoio na defesa contra os animais selvagens e isso pode dever-se ao facto da maioria praticarem agricultura e esta atividade ser um dos catalisadores do conflito Homem-Fauna bravia. Moradores do posto administrativo de Lugenda tem-se beneficiado de um Kit de alimentos, o que não se verifica no posto administrativo de Mecula-Sede. O emprego é também um dos benefícios apontados pelos entrevistados e cobre cerca de $35 \%$ dos entrevistados.

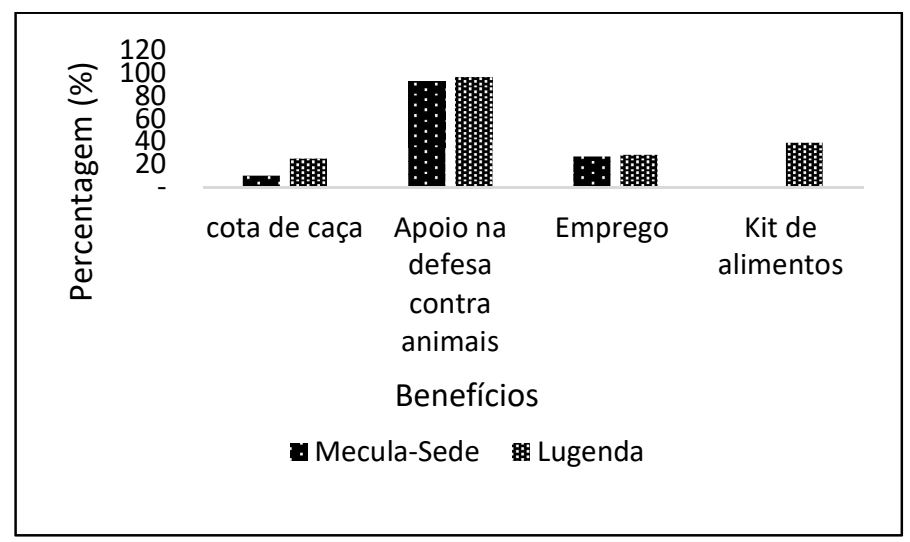

Figura 3: Benefícios aos Membros da Comunidade.

Foi feita uma análise de regressão para verificar possíveis associações entre o número de atividades de maneio e conservação a que cada entrevistado está envolvido e as características socioeconômicas e os resultados estão apresentados na tabela 3. Os anos de escolaridade e o género são as variáveis que mostraram ter influência no número de atividades a que os membros da comunidade estão envolvidos. Os homens têm tendência a participar mais em atividades de maneio e conservação em relação às mulheres e pessoas com um nível de escolaridade mais elevado tendem a adotar as atividades de maneio e conservação. 
Tabela 2: Resultados da Análise de Regressão de Poisson.

\begin{tabular}{|c|c|c|c|c|c|c|c|c|}
\hline \multirow{3}{*}{ Variáveis } & \multicolumn{8}{|c|}{ Posto Administrativo } \\
\hline & \multicolumn{4}{|c|}{ Mecula } & \multicolumn{4}{|c|}{ Lugenda } \\
\hline & Estimativas & Erro pad & valor Z & $\operatorname{Pr}(>|z|)$ & Estimativas & Erro pad & Valor Z & $\operatorname{Pr}(>|z|)$ \\
\hline (Intercept) & -0.359 & 0.406853 & -0.88 & 0.3776 & -0.33667 & 0.297045 & -1.13 & 0.257 \\
\hline Sexo & 0.36238 & 0.173867 & 2.084 & $0.037^{*}$ & 0.621592 & 0.154561 & 4.022 & $0.00 * * *$ \\
\hline Idade & 0.0025 & 0.005841 & 0.428 & 0.6687 & -0.00716 & 0.00413 & -1.73 & 0.083 \\
\hline Agregado & 0.021 & 0.030271 & 0.694 & 0.4879 & -0.02388 & 0.028685 & -0.83 & 0.405 \\
\hline Educação & 0.15149 & 0.030076 & 5.037 & $0.0^{* * *}$ & 0.169026 & 0.024509 & 6.896 & $0.00 * * *$ \\
\hline
\end{tabular}

\section{Projetos de Desenvolvimento Resultantes dos 20\% das Receitas de Exploração dos Recursos}

Na tabela 4, estão apresentados os tipos de projeto aos quais é destinado o valor dos $20 \%$ resultantes da exploração dos recursos e outras atividades como o turismo. O teste de independência mostra haver relação entre o Posto administrativo e tipo de projeto a que são alocados os $20 \%\left(P_{\text {value }}=0.00, \mathrm{GL}=7\right.$, $\left.X_{c a l}=93.18\right)$. O posto administrativo de Lugenda tem mais apostado em projetos ligados à área de saúde processamento de alimentos enquanto o posto administrativo de Mecula aposta mais na área da educação e pecuária.

Tabela 4: Projetos financiados pelos $20 \%$.

\begin{tabular}{|c|c|c|}
\hline \multirow{2}{*}{ Projeto } & \multicolumn{2}{|c|}{ Posto Administrativo } \\
\cline { 2 - 3 } & Lugenda & Mecula-Sede \\
\hline Construção de sala de aulas & $5 \%$ & $16 \%$ \\
\hline Compra de cabritos & $0 \%$ & $56 \%$ \\
\hline Microindústria Processadora de grãos & $20 \%$ & $0 \%$ \\
\hline Cadeiras para reuniões & $7 \%$ & $4 \%$ \\
\hline Esteiras para mesquita & $0 \%$ & $8 \%$ \\
\hline Construção de posto de saúde & $26 \%$ & $0 \%$ \\
\hline Melhoria de vias de acesso & $5 \%$ & $0 \%$ \\
\hline Sem registo & $38 \%$ & \multicolumn{2}{|c|}{$16 \%$} \\
\hline
\end{tabular}

\section{DISCUSSÃO}

A predominância de jovens nos dois postos administrativos (figura 1) pode indicar uma potencial força de trabalho que pode ser aproveitada se for bem explorada. Programas mais direcionados para a educação dos jovens na área de conservação podem trazer bons resultados. Nadeson et al. (2007) enfatizam o papel da educação ao garantir que os jovens adquiram sensibilidade, habilidades e conhecimentos em relação à conservação e às preocupações ambientais. No entanto, existe uma tendência de a população adotar medidas que para além do benefício à conservação, também beneficiam de forma direta aos residentes, que é o caso do controlo de queimadas e a caça ilegal (tabela 2).

As queimadas descontroladas tem tido efeitos negativos na vida das pessoas, pois, por um lado tem ocorrido com muita frequência, o que reduz a produtividade económica das florestas, com implicações negativas no desenvolvimento da riqueza local e por outro lado há o risco de perda de vidas humanas e infraestruturas devido ao fogo (AMADEU, 2015). A caça ilegal também tem implicações na sobrevivência das comunidades pois alguns membros usam a caça para o seu sustento, pelo que, ao haver caçadores ilegais, pode aumentar a pressão sobre os animais selvagens e comprometer a disponibilidade destes animais para 
os moradores locais. Neste sentido, ainda se torna menos claro até que ponto as comunidades residentes dentro da Reserva do Niassa estão envolvidas nas práticas de conservação.

A partir da tabela 3, fica ainda mais clara a importância que a educação tem na adoção de práticas de maneio e conservação. A existência de uma divisão sexual do trabalho em Moçambique, onde o homem fica encarregado das atividades que geram rendimento ou dinheiro e as grandes desigualdades de género nas comunidades rurais (AGY, 2017) podem estar por detrás da fraca participação da mulher nas atividades de maneio e conservação, dado que geralmente poucas mulheres tem participado em reuniões dentro da comunidade, tornando o acesso à informação limitado para as mulheres.

O kit de alimentos que é dado aos residentes de Lugenda pode ter a ver com o facto desta ser uma zona remota com pouco acesso à mercados de venda de produtos alimentares, além de que, de acordo com oferecer alimentos pode ser uma forma particularmente eficaz de aumentar o consumo de micronutrientes, particularmente entre certos grupos-alvo (crianças, mulheres). Contudo, há que se reavaliar a sustentabilidade deste programa para evitar futuros conflitos, uma vez que o kit não tem chegado a todos os membros da comunidade e não estão claros os critérios para a seleção das famílias beneficiárias.

Transferências de dinheiro são cada vez mais usadas na África Subsaariana como um instrumento de proteção social para combater a pobreza e melhorar o bem-estar das comunidades (SKOVDAL, 2013). Pequenas quantias em dinheiro dadas a famílias pobres numa base regular e previsível (frequentemente mensal ou bimensal) permitem o controlo, independência e tomada de decisão (HANLON, 2010; STANDING, 2008).

Contudo, o valor dos $20 \%$ da exploração dos recursos e do turismo é transferido à comunidade numa base anual, e os projetos aos quais são poder não ser a melhor forma de utilizar este dinheiro, pois era suposto usarem o dinheiro para projetos que beneficiem a comunidade no geral, mas este só beneficia as pessoas com certo nível de poder dentro da comunidade. A compra de cabritos só beneficia aos líderes e aos membros da comunidade com poder político pois estes é que, na maioria das vezes, tem recebido boa parte destes animais para o benefício próprio.

A compra de esteiras para a mesquita só beneficia a parte muçulmana da comunidade, fazendo com que os praticantes doutras religiões fiquem revoltados. As transferências de renda, que visam algumas famílias e não outras, podem falhar e causar conflitos, ciúmes e outras consequências não intencionais dentro das comunidades (SKOVDAL, 2013), pelo que se torna necessária uma revisão do modelo atual usado na atribuição deste valor por forma a garantir igualdade e maior percepção dos benefícios por parte de todos os membros da comunidade.

\section{CONCLUSÕES}

Baseado nos resultados, conclui-se que há pouco envolvimento dos membros das comunidades nas atividades de maneio e conservação que não tem um benefício direto sobre as suas vidas, em particular. Contudo, membros da comunidade com grau de escolaridade mais elevado tendem a adotar mais as práticas de conservação. 
Os benefícios da conservação não são perceptíveis para toda população das comunidades estudadas, uma vez que apenas os cidadãos com certo grau de poder, como líderes comunitários e membros com alguma força política é que tem aproveitado maior parte dos benefícios, principalmente o valor correspondente aos $20 \%$ da exploração de recursos, criando-se desigualdades na partilha destes benefícios.

\section{REFERÊNCIAS}

ADAMS, W. M.. Biodiversity conservation and the eradication of poverty. Science, New York, v.306, n.5699, p.1146-1149, 2004

AGY, A. R.. Desigualdades de género em contextos rurais em Moçambique: estudos de caso em localidades na Província de Nampula. Maputo: IESE, 2017.

AMADEU, L. O.. Avaliação da Incidência de Queimadas no Ecossistema de Mopane no Distrito de Mabalane de 2002 a 2011. Maputo: 2015

BALMFORD, A.. Economic Reasons for Conserving Wild Nature. Science, v.297, n.5583, p.950-953, 2002.

BATISTA, D. T.. Modelos para dados de contagem com superdispersao: uma aplicacao em um experimento agronomico. Piracicaba: 2015.

DISTA, N.; NHANCALE, B. A.. Maneio Comunitários dos Recursos Naturais e Desenvolvimento de Pequenas e Médias Empresas Florestais, p.1-22, 2009.

FERNANDES-BACA, J.; MARTIN, A. S.. Indigenous peoples and Protected Areas Management: Innovations in Conservation Series, Parks in Peril Program. Park in Pểil: Innovations in Conservation Series, p.1-20, 2007.

GRIFFITHS, T.. Indigenous Peoples' experiences of GEFfunded Biodiversity Conservation: a critical study. Moretonin-Marsh: 2005.

HANLON, J.. Just give money to the poor. In: International Congress of the Basic Income Earth Network, 13. Annals. Bergen: 2010.

MATOS, E.; MEDEIROS, R.. Conservação e Desenvolvimento de Comunidades Tradicionais: o caso de Chimanimani no centro de Moçambique. Rio de Janeiro: UERJ, 2010.

NADESON, T.; BARTON, M.. The Role of Youth in the
Conservation of Biodiversity: WWF-Malaysia's Experiences. Kuala Lumpur, 2007.

NHANTUMBO, I.; MACQUEEN, D.. Direitos das Comunidades Direitos das Comunidades: realidade ou retórica?. Maputo: 2003.

NHONGO, E. J. S.. Caracterização fenológica da cobertura vegetal com base em série temporal NDVI / MODIS na Reserva do Niassa - Moçambique: Phenological Characterization of Vegetation Cover based on Time Series of NDVI / MODIS, in Niassa Reserve-Mozambique. Revista Brasileira de Cratografia, p.1175-1187, 2017.

ORAM. Associação Rural de Ajuda Mútua. O Impacto da politica agrária em Moçambique. Maputo: ORAM, 2010.

PRODEZA. Projeto de Apoio ao Desenvolvimento Rural na Província de Zambézia. Actividades dos Recursos Naturais na Zambézia. Zambezia: 2009.

SCHERL, L. M.. As áreas protegidas podem contribuir para a redução da pobreza?: Oportunidades e limitações. Gland: 2006.

SILVA, C.. Áreas Protegidas em Portugal: que papel? Conservação versus desenvolvimento. Revista Geolnova, n.2, p.27-44, 2000.

SKOVDAL, M.. Social acceptability and perceived impact of a community-led cash transfer programme in Zimbabwe. BMC Public Health, v.13, p.342, 2013.

STANDING, G.. How Cash Transfers Promote the Case for Basic Income. Basic Income Studies, v.3, n.1, 2008.

STEARMAN, A. M.. On Common Ground: the Nature Conservancy and Traditional Peoples: the Rio Chagres, Panama Workshop. Arlington: Nature Conservancy, 1996. p.267.

A CBPC - Companhia Brasileira de Produção Científica (CNPJ: 11.221.422/0001-03) detém os direitos materiais desta publicação. Os direitos referem-se à publicação do trabalho em qualquer parte do mundo, incluindo os direitos às renovações, expansões e disseminações da contribuição, bem como outros direitos subsidiários. Todos os trabalhos publicados eletronicamente poderão posteriormente ser publicados em coletâneas impressas sob coordenação da Sustenere Publishing, da Companhia Brasileira de Produção Científica e seus parceiros autorizados. Os (as) autores (as) preservam os direitos autorais, mas não têm permissão para a publicação da contribuição em outro meio, impresso ou digital, em português ou em tradução. 